\title{
Reliability Evaluation and Prediction of Low Voltage Distribution Network
}

\author{
Xiaojun Pan ${ }^{1, a}$, Aihong Tang ${ }^{1, b}$, Huiyuan Yang ${ }^{1, \mathrm{c}}$ and Chao $\mathrm{Li}^{1, \mathrm{~d}}$ \\ ${ }^{1}$ School of Automation,Wuhan University of Technology, China \\ a1552991809@qq.com, b375943243@qq.com, 416518269@qq.com, d10136109@qq.com
}

Keywords:reliability ,forward and backward substitution method ,measures

\begin{abstract}
Forward and backward substitution method has been used as the power flow calculation method of any low voltage distribution network, and therefore utilization rate of electrical energy of the lines, transformer and line loss are obtained. These are the datum to assess the reliability of low voltage distribution system, and to reflect the level of reliability of power supply of the whole distribution system. At the same time, reliability indices of the low voltage distribution network are predicted by the method of probability and statistics. According to the reliability evaluation and prediction of the distribution network, the weak link of the distribution system is found and scientific decision-making basis is provided for the construction and operation management of distribution system.
\end{abstract}

\section{Introduction}

With steady economic development of our country and improvement of people's living standard, people depend more and more on the power supply, the society requests higher reliability of power supply. According to the voltage level, distribution network can be divided into high voltage distribution network $(35 \sim 110 \mathrm{kV})$, medium voltage distribution networks $(6 \sim 10 \mathrm{kV}, 20 \mathrm{kV})$ and low voltage power distribution network $(220 \sim 380 \mathrm{~V})[1]$. Low voltage distribution network directly facing the terminal user is an important part of the distribution network. Therefore, it is significant to make clear the weak link and to put forward the improvement measures through reliability evaluation and prediction of low voltage distribution network.

\section{Evaluation for Existing Network}

The definition of evaluation for existing network is that according to the parameter of transformers, wires in low voltage distribution network and topological graph of specific area for power flow calculation, the load rate of transformers, lines and line loss are obtained so that we can evaluate the network of the specific area by these datum. The following figure 1 as an example of distribution network is used to introduce the power flow calculation with forward and backward substitution method[2].

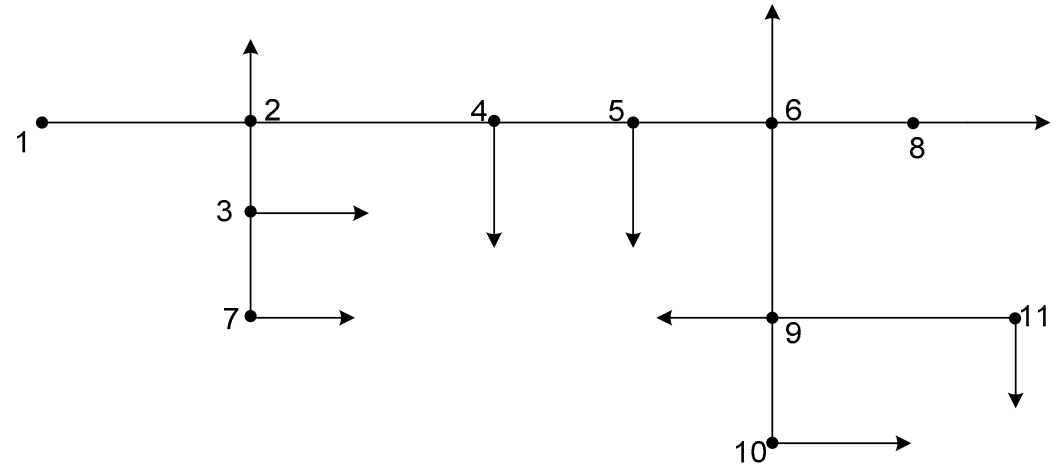

figure 1 topological graph of a distribution network

The network topology is entered based on Excel form, the input format is shown in table 1: 
Table 1 data input format

\begin{tabular}{|c|c|c|c|c|c|c|c|}
\hline $\begin{array}{c}\text { Line } \\
\text { number }\end{array}$ & $\begin{array}{c}\text { Initial } \\
\text { node }\end{array}$ & $\begin{array}{c}\text { End } \\
\text { node }\end{array}$ & $\begin{array}{c}\text { Branch } \\
\text { resistance }\end{array}$ & $\begin{array}{c}\text { Branch } \\
\text { reactance }\end{array}$ & $\begin{array}{c}\text { Node } \\
\text { number }\end{array}$ & $\begin{array}{c}\text { Load } \\
\text { active } \\
\text { power }\end{array}$ & $\begin{array}{c}\text { Load } \\
\text { reactive } \\
\text { power }\end{array}$ \\
\hline 1 & 1 & 2 & $\mathrm{XXX}$ & $\mathrm{XXX}$ & 1 & $\mathrm{XXX}$ & $\mathrm{XXX}$ \\
\hline 2 & 2 & 3 & $\mathrm{XXX}$ & $\mathrm{XXX}$ & 2 & $\mathrm{XXX}$ & $\mathrm{XXX}$ \\
\hline 3 & 2 & 4 & $\mathrm{XXX}$ & $\mathrm{XXX}$ & 3 & $\mathrm{XXX}$ & $\mathrm{XXX}$ \\
\hline 4 & 3 & 7 & $\mathrm{XXX}$ & $\mathrm{XXX}$ & 4 & $\mathrm{XXX}$ & $\mathrm{XXX}$ \\
\hline 5 & 4 & 5 & $\mathrm{XXX}$ & $\mathrm{XXX}$ & 5 & $\mathrm{XXX}$ & $\mathrm{XXX}$ \\
\hline 6 & 5 & 6 & $\mathrm{XXX}$ & $\mathrm{XXX}$ & 6 & $\mathrm{XXX}$ & $\mathrm{XXX}$ \\
\hline 7 & 6 & 8 & $\mathrm{XXX}$ & $\mathrm{XXX}$ & 7 & $\mathrm{XXX}$ & $\mathrm{XXX}$ \\
\hline 8 & 6 & 9 & $\mathrm{XXX}$ & $\mathrm{XXX}$ & 8 & $\mathrm{XXX}$ & $\mathrm{XXX}$ \\
\hline 9 & 9 & 10 & & & 9 & $\mathrm{XXX}$ & $\mathrm{XXX}$ \\
\hline 10 & 9 & 11 & & & 10 & & \\
\hline & & & & & 11 & & \\
\hline
\end{tabular}

This topological graph is a radial network for power supply which is from a power supply point to any load point, a radial network is a tree network. Power supply point is called the root node, the node with which only one branch is connected is called the leaf node. When the forward and backward substitution method is used for power flow calculation, the branch current is calculated according to the current path of the leaf node to the root node and the voltage of each node is forward calculated according to the relationship between the upstream and downstream nodes. In this process, we need the help of the leaf node to the root node matrix and the downstream node matrix. The following is the introduction of the two matrices:

\subsection{Definition}

In the distribution network topology, node 1(low-voltage outlet of the transformer) is called root node, nodes 7,8,10,11 are called leaf nodes.

The leaf node to the root node matrix: An $\mathrm{E} \times \mathrm{N}$ array, $\mathrm{E}$ is the number of leaf nodes, $\mathrm{N}$ is the number of nodes of network. The initial value of all the elements of the array is 0 . We can go back from the leaf nodes to root node to find the shortest path through nodes according to the relationship of connection between nodes. The number of leaf nodes is equal to the number of loops and the number of rows of the leaf node to the root node matrix.

The leaf node to the root node matrix from figure 1 is shown in table 2:

Table2 leaf node to the root node matrix

\begin{tabular}{|c|c|c|c|c|c|c|c|c|c|}
\hline 7 & 3 & 2 & 1 & 0 & 0 & 0 & 0 & 0 & 0 \\
\hline 8 & 6 & 5 & 4 & 2 & 1 & 0 & 0 & 0 & 0 \\
\hline 10 & 9 & 6 & 5 & 4 & 2 & 1 & 0 & 0 & 0 \\
\hline 11 & 9 & 6 & 5 & 4 & 2 & 1 & 0 & 0 & 0 \\
\hline
\end{tabular}

Downstream node matrix: Starting from root node, the node numbers are filled in the first column of downstream node matrix in order. The numbers of downstream nodes which is directly connected to node number of each row of table 1 are successively filled in each column of downstream node matrix corresponding to the row. Downstream node matrix is a $\mathrm{N} \times \mathrm{M}$ array( $\mathrm{M}$ represents the maximum number of branch points). The initial value of all the elements of the array is 0 . The matrix represents the relationship between the upstream and downstream nodes in the topology. 
Table3 Downstream node matrix

\begin{tabular}{|c|c|c|}
\hline 1 & 2 & 0 \\
\hline 2 & 3 & 4 \\
\hline 3 & 7 & 0 \\
\hline 4 & 5 & 0 \\
\hline 5 & 6 & 0 \\
\hline 6 & 8 & 9 \\
\hline 7 & 0 & 0 \\
\hline 8 & 0 & 0 \\
\hline 9 & 10 & 11 \\
\hline 10 & 0 & 0 \\
\hline 11 & 0 & 0 \\
\hline
\end{tabular}

\subsection{Mathematical Models and Method}

The leaf node to the root node matrix: The leaf node number is determined according to table 1 . The leaf nodes are only among the end nodes, so the nodes which belong to the third column not to the second column are the leaf nodes when the elements in the third column is compared with the elements in the second column of the table 1. Then all the leaf nodes are found and placed in the first column of the leaf node to the root node matrix. The row number of each leaf node is found in the column of end nodes, then the node number is found in the column of initial node according to corresponding row number. For example, the node 7 corresponds to node 3 which is an initial node, then node 3 is placed in the second column of the row in which node 7 is of the leaf node to the root node matrix. Find the row number of node 3 in the end node column again and find the corresponding initial node. At last, place it in the third column of the row in which node 7 is of the the leaf node to the root node matrix. The rest can be done in the same way.

Downstream node matrix: Start from number 1, the row with the same number in the second column of table 1 is found, then the corresponding number in the third column is filled in the first row of the second column of downstream node matrix. If there still exists the number 1 node, the node number will be filled in each column of the first row of the downstream node matrix until there is no number 1 existing in the second column of table 1. According to the method mentioned above, the numbers are filled in from 1 to $\mathrm{N}$ one by one, and at the same time downstream node matrix can be generated.

Power flow calculation of low voltage distribution network with the forward and backward method.

(1)The first round of iteration, each node of the network is assigned rated value $220 \mathrm{~V}$ (the phase angle is taken as 0 ).

(2)Look up wire impedance value per $\mathrm{km}$ and current carrying capacity according to material of wire.

(3)According to the datum of every kilowatt hour meter, the active power $\mathrm{P}$ is obtained, then the reactive power can be calculated with power factor $\cos \varphi=0.85$. According to the leaf node to the root node matrix, calculate forward the current of each branch with the formula (1)[3].

$$
\dot{I}=\frac{P-j Q}{U_{N}}
$$

When program comes to bifurcation node(according to the downstream node matrix), whether the current of line connected to the node has been calculated is first to be determined. If the calculation is completed, we will continue to calculate the current to the root node, if not, we will need to wait until current of all connected lines are worked out. Then calculate the current.

(4)With the branch current that has been calculated in step 3 and the known transformer secondary voltage (generally the rated voltage, phase angle is set to 0 ), the voltage of node is calculated one by one according to the the leaf node to the root node matrix and downstream node matrix. At last, the voltage of each node is worked out by the formula (2). 


$$
\left|U_{b}^{n}-U_{b}^{n-1}\right| \leq \varepsilon
$$

(5)Do the iteration for $\mathrm{n}$ times with program of algorithm until $\dot{U}^{\prime}=\dot{U}-\dot{I}(R+j X) \quad\left(\mathrm{U}_{\mathrm{b}}{ }^{\mathrm{n}}\right.$ is the voltage of the node after iteration for $n$ times, $\mathrm{U}_{\mathrm{b}}{ }^{\mathrm{n}-1}$ is the voltage of the node after iteration for $\mathrm{n}-1$ times, $\varepsilon$ is a set value )[4].Then the voltage that has been calculated could be regarded as the actual voltage of the node.

(6)The utilization rate of electrical energy of users can be obtained by the formula (3)

$$
\text { utilization rate }=\frac{\text { the actual measured value }}{\text { the rated value }}
$$

(7)Line loss of each line can be calculated by the formula (4)

$$
S=I^{2}(R+j X)
$$

\section{Reliability Prediction}

Reliability prediction is a method to calculate the reliability indices of a specific network with the use of the known failure rate and repair rate of component.

3.1 Reliability indice

According to current system from IEEE Guide for Electric Power Distribution Reliability Indices, reliability indices are divided into main indices and reference indices. Here are 5 main indices[5]: SAIFI:System Average Interruption Frequency Index. The formula is shown as (5).

$$
\text { SAIFI }=\frac{\sum \text { Total Number of Customers Interrupted }}{\text { Total Number of Customers Served }}
$$

SAIDI: System Average Interruption Duration Index.The formula is shown as (6).

$$
S A I D I=\frac{\sum(\operatorname{Re} \text { storation Time for Each Interruption Event } \times \text { Number of Interrupted customers })}{\text { Total Number of Customers Served }}
$$

CAIDI: Customer Average Interruption Duration Index.The formula is shown as (7).

$$
C A I D I=\frac{\sum(\text { Re storation Time for Each Interruption Event } \times \text { Number of Interrupted Customers })}{\text { Total Number of Customers Interrupted }}
$$

CAIFI: Customer Average Interruption Frequency Index. The formula is shown as (8).

$$
\text { CAIFI }=\frac{\sum \text { Total Number of Customer Interruptions }}{\text { Total Number of Distinct Customers Interrupted }}
$$

ASAI: Average Service Availability Index. The formula is shown as (9).

$$
A S A I=\frac{\text { Customers Hours Service Availability }}{\text { Customers Hours Service Demand }}
$$

The traditional methods for quantitative prediction of the reliability of distribution system are mainly divided into two categories, namely, analytical method and simulation method. Here we choose to combine sequential Monte Carlo method of simulation method[6] with fault tree analysis. The following are steps:

(1)Each line is simulated based on the topology of the graph. It is assumed that each line is in the operating state and the sampling value of state duration that obeys exponential distribution is 
obtained by the formula (10).

$$
D_{i}=\frac{1}{\lambda_{i}} \ln \mathrm{R}_{i}
$$

In the formula, $R_{i}$ is a random number corresponding to the element $i$ in the $[0,1]$ range of uniform distribution. If the current state is running, $\lambda_{i}$ is the failure rate of the components, and if the current state is closed, it is the repair rate of the element.

(2)With the use of failure rate and repair rate of each line and the random number of $[0,1]$ range, the running time and outage time of each line can be obtained. Through the simulation of the first round, the time sequence graph of different line (the graph is shown in figure 2) can be drawn. The line which costs the shortest running time in the first round of the simulation can be found, which is the first line of fault. Count the number of users which is influenced by the line fault with fault tree analysis[7].
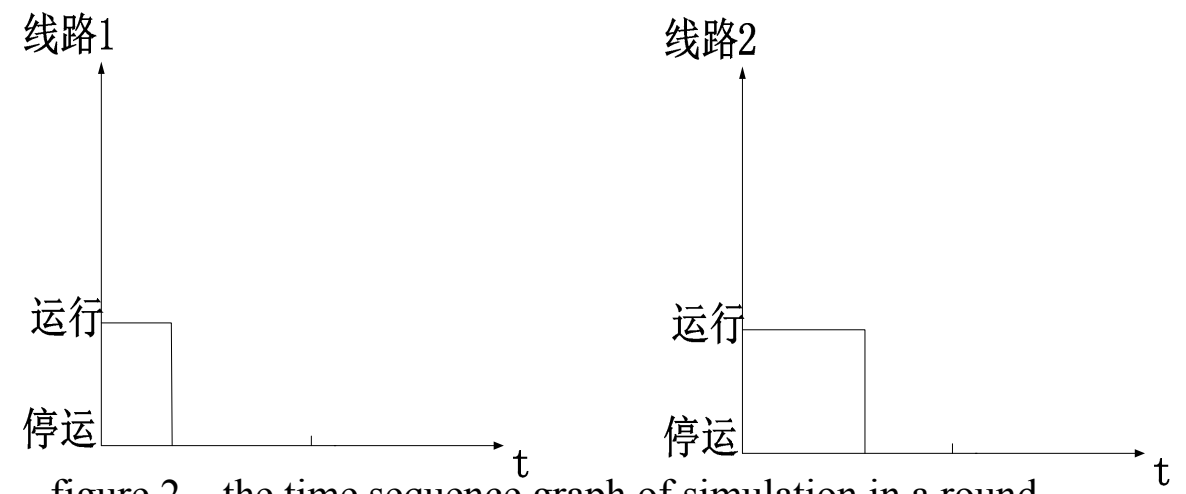

(3)Stop simulation until time is equal to 500 years after several rounds of simulation. Indices such as SAIFI, SAIDI, CAIDI, CAIFI, ASAI can be calculated by(5), (6), (7), (8), (9), according to the number of users and the interruption duration of the time sequence graph of simulation.

\section{Measures to Improve the Reliability of Low Voltage Distribution Network}

Finding the weakness according to the reliability evaluation and prediction of distribution network and putting forward the corresponding improvement measures is our fundamental objective to do research on the reliability of low voltage distribution network. The following is the introduction of several methods used to improve the reliability of the low voltage distribution network.

4.1 An increase in reactive power compensation

Low voltage distribution network with three kinds of compensation method[8]

(1)Low voltage centralized compensation

Concentrated compensation is on the $380 \mathrm{~V}$ side of transformer. The compensation device is usually the low voltage shunt capacitor cabinet controlled by microcomputer. The corresponding number of capacitors are put into to track and compensate according to the fluctuation of load level.

(2)Reactive compensation on the pole

Install the outdoor $10 \mathrm{kV}$ shunt capacitor on the overhead line tower (or set up other poles)for reactive power compensation.

(3)reactive power compensation for the user side

Direct reactive power compensation for the terminal users will be the most appropriate way to reduce the power loss and maintain the network voltage level.

Active power, reactive power loss of transmission line of three phase alter current can be calculated by formula(11),(12).

$$
\Delta P_{L}=3 \times r_{0} \times\left(\frac{W_{H}}{\sqrt{3} \times U_{H} \times \cos \varphi_{L}}\right)^{2} \times t \times 10^{-3}
$$




$$
\Delta Q_{L}=3 \times x_{0} \times\left(\frac{W_{H}}{\sqrt{3} \times U_{H} \times \cos \varphi_{L}}\right)^{2} \times t \times 10^{-3}
$$

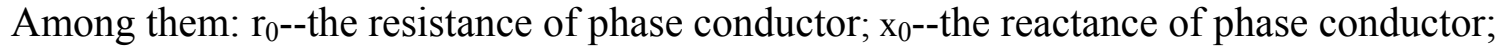
$\mathrm{W}_{\mathrm{H}^{-}}$-the active energy; $\mathrm{U}_{\mathrm{H}^{--}}$the rated line voltage; $\varphi_{\mathrm{L}}$--the power factor angle; $\mathrm{t}$-- running time of line;

For example, if a distribution line loss rate is $10 \%$, the power factor is increased from 0.8 to 0.95 . According to the formula, the line loss rate reduced by $2.91 \%$.

4.2 Determine a reasonable position of a power supply and radius of power supply

The allowable radius of power supply can be obtained according to the offset of voltage and line loss that are allowed. The formula of offset of voltage and line power loss is shown as (13),(14).

$$
\begin{gathered}
\Delta U \%=\frac{\Delta U}{U_{N}}=\frac{P L(r+x \tan \varphi)}{U_{N}^{2}} \times 100 \% \\
\Delta P \%=\frac{\Delta P}{P}=\frac{\frac{S^{2}}{U_{N}^{2}} \times r L}{S \cos \varphi}=\frac{S r L}{U_{N}^{2} \cos \varphi} \times 100 \%
\end{gathered}
$$

In the formula:r is the resistance per unit length, $\mathrm{x}$ is reactance per unit length, $\varphi$ is the power factor angle, $\mathrm{L}$ is radius of power supply.

At the same time, the reasonable radius of power supply can be obtained according to voltage grade and load rate(the radius of 400v power line in the urban area should not be longer than 500 meters). 4.3 Plan the grid structure rationally

"Hand in hand"ring network power supply line should be used, which would greatly improved the lack of power supply. The biggest advantage of it is to distribute energy rationally, shorten the radius of power supply, and thoroughly improve the power quality of the terminal user. Two low voltage lines are connected by a contact switch. When an error occurs on either part of the line, the failure part can be separated in a short time and power will be provided to users without fault from the both sides of switch. So it can not only avoid power blackouts accident, shorten the time of fault repairs, but will also reduce the outage loss, ensure the reliability of using electricity maximally.

4.4 Take full consideration of demand of system load growth, choose the conductor section rationally[9].

The resistance of a wire is inversely proportional to the cross-sectional area, so it is better to choose the conductor with bigger cross-sectional area, in the circumstances power loss can be reduced. On the other hand, thick wire costs more. So both cost and power loss should be taken into consideration.

In grid of $0.4 \mathrm{kv}$, we usually choose the wire with cross-sectional area larger than 70 square millimeters, and the straight and trunk line should be more than 50 square millimeters. When choosing the low voltage wire, we should also take voltage loss into consideration. Furthermore, the connection mode of low voltage network also plays a vital role of line loss, and the point outward radiation should be adopted according to some researches.

4.5 Renovation of the transformer

To transformers in the city, they are lightly loaded in the daytime, while heavily loaded at night. However, the transformers in the countryside are heavily loaded in busy farming season such as "winter pouring" or "summer pouring", but in the other time, they are often lightly loaded even unloaded. As a result, we can not just use transformers with large capacity[10]. Instead, the type of transformers should be chosen according to the facts, which avoids low usage of transformers and reduces the line loss to some extent.

At the same time, we would better choose the type of amorphous alloy iron core or energy-saving 
type, which can reduce the grid loss greatly. Absolutely, the better comprehensive performance they have, the higher prices they are at, so the transformers should be chosen according to the load characteristic in order to ensure the economical efficiency and feasibility of the system.

4.6 Three-phase load balancing of the transformer

Management measures

(1)The establishment of assessment method and system of the three-phase load balancing is used to make workers pay close attention to the changes of three-phase load. The operation condition of transformer is mastered timely through the real-time monitoring of three-phase load[11].

(2)Reduce random access of the single-phase load, enhance the data management.

Technical measures

(1)The network of direct neutral grounding is increased to avoid neutral point deviation because of three-phase load imbalance.

(2)The cross-sectional area of the wire of neutral line is increased to as large as of the phase line to reduce the possibility of line broken.

\section{Summary}

Forward and backward method used in this paper is adapted to the circumstance that ratio of the resistance and reactance is large, which is suitable for power flow calculation of low voltage distribution network. Prediction method is that the combination of the non sequential Monte Carlo method and fault tree method to obtain the reliability indices of distribution network. At last, corresponding measures are selected from the general measures to improve reliability of the actual grid.

\section{Acknowledgements}

This work was financially supported by the National Nature Science Foundation (51177114), Wuhan Applied Basic Research Projects (2014010101010012).

\section{References}

[1]Tingting Xiang:Study on the method of planning of low voltage power distribution network. College of Electrical Engineering,Chongqing University,2012

[2]Chen Gu,Xiufan Yue,Xiaoming Zhang:Power System Protection and Control. Vol.38(2010),p.160-161

[3]Hui Yang,Fushuan Wen,Yongqiang Liu,Peng Liu: Proceedings of the CSU-EPSA .Vol.22(2010),p.123-127

[4]Yangzan He,Zengyin Wen:Analysis of Electric Power System(Huazhong University of Science and Technology Press, Wuhan 2002)

[5]Zhengsen Tang:Research On Improving the Reliability of Measures of Distribution Network of User.College of Electrical and Information Engineering,Changsha University of Science and Technology,2009

[6]Xing Zheng:Evaluation of Distribution Network Evaluation Based On Monte Carlo Method.School of Electrical and Electronic Engineering,Huazhong University of Science and Technology,2011

[7]Kaigui Xie,Tian Xia,Bo Hu,Kan Cao:Sichuan Electric Power Technology.Vol.32(2009),p2-4

[8]Yiyi Wang:Guangdong Electric Power.Vol.120(2007),p.128-129

[9]Kaixuan Zhu:China Electric Power Education.NO.6(2011), p.128-129

[10]Ming Pan:Researeh on Line Loss Reduetion Measures for HaikouLing Shan Rural Medium-low-voltage Distribution Network.School of electrical and Electronic Engineering,North 
China Electric Power University,2010

[11]Hongbin Li,Linyun Chen:Nong Cun Dian Gong.Vol.22(2014),p.34 